Revised- April 21, 2016

\title{
Errors In Bladder Catheterization: Are Residents Ready for Complex Scenarios?
}

${ }^{a}$ Department of Surgery, School of Medicine and Public Health, University of Wisconsin-Madison 750 Highland Avenue, Madison, WI 53726, USA

Corresponding Author:

Bridget O'Connell-Long, BS

Department of Surgery

University of Wisconsin Hospital and Clinics

600 Highland Ave.

K6/135 CSC

Madison, WI 53792

(262) 470-7855

boconnelllon@wisc.edu

Subject Category: Education

\section{Author Contributions:}

Bridget O'Connell-Long performed data analysis and interpretation, writing the manuscript, and critical revision of the manuscript with significant contribution and assistance from Rebecca Ray. Rebekah Fiers contributed to data collection, data analysis and interpretation, and critical revision of the manuscript. Dr. Jay Nathwani contributed to data analysis and interpretation and critical revision of the manuscript. Dr. Carla Pugh performed duties of a senior author; contributed to study conception and design; data collection, analysis and interpretation; writing the manuscript and critical revision of the manuscript

Sources of Funding: Funding for this study came from the National Institutes of Health grant T35DK062709 and the Department of Defense grant W81XWH-13-1-0080. Neither funding source had involvement in study conception or design, data analysis, writing of the manuscript, or decision to submit the article to publication. 


\begin{abstract}
Background

The aim of this study was to investigate whether junior surgical residents had successfully mastered bladder catheterization. Our hypothesis was that surgical residents would be overly confident in their abilities and underestimate the potential for case complexity.
\end{abstract}

\title{
Materials and methods
}

PGY 2-4 surgery residents ( $n=44)$ were given 15 minutes to complete three of four bladder catheterization simulations. Participants reported their mastery by rating confidence using a 5-point Likert scale. Multiple linear regression analysis was used to test predictors of procedure performance.

\section{Results}

Participants made a total of 228 errors with an average of 5.1 errors $(\mathrm{SD}=2.6)$ per participant. The most common errors included not maintaining the sterile field (52.0\%), failure to get urine return (20.3\%), and inflating the catheter balloon before urine return (8.4\%). Some residents committed the same error more than once.

Pre-simulation confidence ratings ranged from " 1 " Not Confident to "5" Extremely Confident. Average pre-simulation confidence was 4.42 (range 1 to $5, \mathrm{SD}=0.85$ ). Sixteen (36\%) residents ranked their presimulation confidence in problem-solving abilities as "Moderately Confident" or below, while twentyeight (64\%) were "Very Confident" or above. The lower the resident's pre-simulation confidence in problem-solving, the more errors they committed during the simulation (beta=-0.33, $\mathrm{t}=-2.15, \mathrm{p}=0.04$ ).

\section{Conclusions}

The residents did not perform as well as they anticipated when presented with more complicated bladder catheterization scenarios. Simulation can be used to identify and expose potential errors that may occur during complex presentations of basic procedures. This type of training and assessment may facilitate mastery.

Keywords: Simulation, Performance, Confidence, Surgery, Education 


\section{Introduction}

Catheter-associated urinary tract infections (CAUTI) are currently the number one hospital-acquired infection in the United States. The CDC estimates that CAUTI attributes to 13,000 deaths and at least $\$ 400$ million in additional cost per year nationally. The detrimental effects of CAUTI have caused the $\mathrm{CDC}$ to define a set of Core Prevention strategies with high-level evidence for prevention. Proper training of personnel and maintaining sterile insertion technique are included in the Core Prevention strategies as defined by the CDC [1-3]. Although nurses perform a majority of urinary bladder catheterizations in the hospital, physicians are often called on for more complex placements. The Association for Healthcare Research and Quality has released a safety program dedicated to the prevention of device-associated infections. The safety program focuses on training resident physicians to be CAUTI-prevention experts, as they are often the first physicians to see the patient [4].

The current body of literature indicates that simulation training can enhance residents' confidence and performance in performing surgical and bedside procedures [5-7]. However, simulation programs can vary widely amongst medical schools and residency training programs, and do not always include complex presentations of procedures for training and assessment [8,9]. In 2007, the ACS/APDS Surgical Resident Skills Curriculum was developed in an attempt to standardize training of surgical residents. According to the ACS/APDS curriculum, urethral catheterization should be mastered during the PGY1 and 2 years [10].

The aim of this study was to investigate whether surgical residents had successfully mastered bladder catheterization. We intended to determine the level of mastery of this basic procedure by presenting more complex and well-known clinical scenarios. Our hypothesis was that surgical residents would be overly confident in their abilities and underestimate the potential for case complexity.

\section{Materials and methods}

\subsection{Setting \& participants}

General surgery residents (PGY 2-4, N=44, 55.6\% female, 44.4\% male) from seven Midwest training programs participated in this study. Surgical residents in their dedicated research years made up $60 \%$ of the participants. Study participants were given 15 minutes to complete three bladder catheterization simulations. Data was captured using motion-tracking software and video and audio recording. Participants were double-gloved using their normal size surgical gloves. Motion tracking wires were secured on their hands between the two gloves (Figure 5). Participants were given no feedback on their performance after completion of the simulation. This study was approved by the University of WisconsinMadison Social and Behavioral Sciences Institutional Review Board.

\subsection{Bladder catheterization simulations}

Study participants completed three of four randomized bladder catheterization simulations as part of a larger study. The simulations represented well-known clinical scenarios: a female trauma patient, a female preoperative patient, a preoperative male with a full urethral blockage, and a male with urinary retention due to benign prostatic hypertrophy (BPH) (Table 1). This study used modified Limbs \& Things@ and Nasco@ bladder catheterization models. The female trauma patient was a standard model that returned bloody urine to indicate prior trauma. The female pre-operative patient simulation was created by adding sutures to the inside of labia to simulate a labial stricture. The penile urethra was completely tied off inside the model to simulate a male with complete urethral blockage. Finally, the penile urethra in male with partial urethral structure was partially obstructed using a polyethylene tube. 
Participants were given a short description of the clinical scenario that corresponded with each simulation. Participants were provided with a simulated catheterization kit including sterile water, lubricant, and a choice of 5 different catheter sizes. The residents were told to assume that the field was sterile and the patient had been prepped. Additionally, participants were given the option to place a urology consult at any point in the simulation.

\subsection{Participant data}

One investigator reviewed video and audio data from each participant and evaluated procedure performance. Errors were defined using the ACS/APDS Technical Skills Curriculum [10]. Investigators used a standard rubric from the ACS/APDS Technical Skills Curriculum to evaluate errors. Error definitions are shown in Figure 3. The residents were asked to complete a survey both pre- and postsimulation. They rated the perceived procedure difficulty and their personal confidence level using a 5point Likert scale for three categories: identifying relevant anatomy, problem solving, and completing the entire surgical task (Figure 1). They also rated the amount of skill reduction they expected as a function of their dedicated time in laboratory research.

\subsection{Data analysis}

Study data were collected and managed using REDCap electronic data capture tools hosted at the University of Wisconsin-Madison, School of Medicine and Public Health [11]. All analyses were performed using SPSS [12]. Multiple linear regression analysis was used to model perceived mastery as predictors of procedure performance as measured by errors and time to call for a urology consult.

\section{Results}

\subsection{Pre-simulation confidence}

Pre-simulation confidence ratings ranged from "1" Not Confident to "5" Extremely Confident. Average pre-simulation confidence was 4.42 (range 1 to $5, \mathrm{SD}=0.85$ ). There was no difference between PGY in self-reported confidence. The lowest PGY average was PGY2 (3.67) and the highest was PGY3 (4.83) Sixteen $(36 \%)$ residents ranked their pre-simulation confidence in problem-solving abilities as "Moderately Confident" or below, while twenty-eight (64\%) were "Very Confident" or "Extremely Confident" (Figure 2). The lower the resident's pre-simulation confidence in problem-solving, the more errors they committed during the simulation (beta=-0.33, $\mathrm{t}=-2.15, \mathrm{p}=.04$ ). Participants with higher presimulation confidence in problem-solving abilities took less time before deciding to place a urology consult $(\mathrm{beta}=-1.53, \mathrm{t}=-4.32, \mathrm{p}=0.001)$.

\subsection{Post-simulation confidence}

Post-simulation confidence ratings ranged from " 1 " Not Confident to " 5 " Extremely Confident. Average post-simulation confidence was 3.56 ( $\mathrm{SD}=0.81$ ), with $3=$ Moderately Confident and $4=$ Very Confident (Figure 1). There was no relationship between post-simulation confidence level and the total errors $(\mathrm{p}>0.05)$.

\subsection{Errors}

Participants made a total of 228 errors with an average of 5.1 errors $(\mathrm{SD}=2.6)$ per participant. The average number of errors per participant were 1.95 for female trauma, 1.29 for female post-op, 1.97 for the male with partial urethral blockage, and 1.73 for male with complete urethral blockage (Figure 2). The most common errors included not maintaining the sterile field (52.0\% of errors), failure to get urine return (20.3\% of total errors), and inflating the catheter balloon before urine return (8.4\% of total errors) (Figure $3)$. Residents who perceived a greater reduction in technical skills during their dedicated research years made fewer errors (beta $=-0.42, \mathrm{t}=-2.76, \mathrm{p}=0.01)$. Twenty-three $(53.3 \%)$ of residents failed to inflate the 
catheter balloon after urine return, 18 (40.0\%) failed to maintain the sterile field, and $14(33.3 \%)$ did not use lubricant (Figure 4). Examples of common errors are shown in Figure 5.

\section{Discussion}

This study investigated how well surgical residents had mastered urinary bladder catheterization. We found that residents did not perform as well as they anticipated when presented with more complicated bladder catheterization scenarios. We assumed that if surgical residents had reached a level of mastery, we would see almost no errors during the simulation. Our data indicates they have not mastered this basic, high-volume procedure despite relatively high confidence levels prior to the simulation. Additionally, the most common errors seen during the simulation (Figure 3) have been shown to contribute directly to the development of catheter-associated urinary tract infections (CAUTI) [2,3].

Our study found that the most common error amongst residents was failure to maintain the sterile field. We found that $40 \%$ of participants committed this error, indicating that this is not a result of multiple errors by a few individuals. These results are significant as maintaining sterile insertion technique shows high-level evidence for the prevention of CAUTI. Two other common errors amongst study participants could contribute to increased risk of infection: inflating the catheter balloon before urine return and inflating the balloon after bloody urine return (Figure 4). Inflating the balloon before placement in the bladder can cause trauma to the urethral tissue and facilitate the spread of bacteria into the bloodstream. Additionally, the bloody urine indicates previous trauma and warrants a consult and potential removal of the indwelling catheter. According to the New England Journal of Medicine guidelines, known or suspected urethral injury is an absolute contraindication to urethral catheterization [13]. Our results indicate that surgical residents may be unaware or non-compliant with these established guidelines regarding insertion of urinary catheters.

This study is limited by our ability to use simulation to measure clinical performance, as participants' behavior during a simulated procedure may differ from the clinical setting. While participants were instructed to treat the simulation as a real-life patient and environment, performance anxiety, video recording and motion-tracking equipment, competition amongst residents, and the presence of the researchers could have influenced the behavior of our study participants. These factors may limit our ability to generalize this study to real-life performance. Due to this, is difficult to determine if the most common errors amongst residents in our study are an accurate reflection of those made in a clinical setting. However, many clinical and academic settings have similar distractions, and we still expect surgical residents to have reached a level of automaticity for this procedure where they would be unaffected by these distractions.

One potential barrier to mastery is the amount of repetition that junior residents are exposed to during their training. Since urinary catheterization has primarily become a nursing procedure, residents may not perform the volume of procedures that are needed in order to adequately assess and problem-solve more complex presentations [14]. Our results show that $36 \%$ of junior residents reported "Moderate Confidence" or below with respect to problem-solving for urinary catheterization. As expected, we found that lower pre-simulation confidence in problem-solving resulted in a higher number of errors during the simulation. However, our study also found that residents who perceived a greater reduction in technical surgical skills during their dedicated research years made fewer errors during the bladder catheterization simulation. This may mean they were more careful during the simulation as a result of believing that they had lost some technical skills. These findings support the_need to develop standardized and concrete skills assessments to ensure that all junior residents have mastered their skills curriculum. Using simulation to assess resident performance could help reduce patient morbidity and cost related to unnecessary errors. 


\section{Conclusions}

The results of this study demonstrate a need to develop a culture in surgery where residents are able to address gaps in knowledge and practice basic procedures until they reach a level of mastery. Simulation is a valuable tool that allows us to identify areas for improvement without the risks to patients. Additionally, simulation can be used to help residents identify and expose potential errors that may occur during complex presentations of basic procedures. This type of training and assessment is necessary and can facilitate mastery.

\section{Acknowledgements}

I would like to acknowledge Shannon DiMarco for contributions to data collection and critical revision of the manuscript. 
Fig. 1 Resident pre-simulation confidence in problem-solving ability for urinary catheterization

Fig. 2 Average number of errors per resident for each bladder catheter simulator

Fig. 3 Composition of errors committed by surgical residents across all four models

Fig. 4 Percent of surgical residents committing the most common errors

Fig. 5 Example of errors in maintaining the sterile field 


\section{References}

[1] Chenoweth C, Saint S. Preventing Catheter-Associated Urinary Tract Infections in the Intensive Care Unit. Crit Care Clin 2013;29:19-32. doi:10.1016/j.ccc.2012.10.005.

[2] Gould C V, Umscheid C a, Agarwal RK, Kuntz G, Pegues D a, Patrick J. Guideline for Prevention of Catheter - Associated Uriinary Tract Infections 2009. Hicpac 2009:1-67. doi:10.1086/651091.

[3] Cochran S. Care of the indwelling urinary catheter: is it evidence based? J Wound, Ostomy, Cont Nurs 2007;34:282-8. doi:10.1097/01.WON.0000270823.37436.38.

[4] for Healthcare Research A. AHRQ Safety Program for Reducing CAUTI in Hospitals Implementation Guide 2 n.d.

[5] Cooke JM, Larsen J, Hamstra SJ, Andreatta PB. Simulation enhances resident confidence in critical care and procedural skills. Fam Med 2008;40:165-7.

[6] Smith CC, Huang GC, Newman LR, Clardy PF, Feller-Kopman D, Cho M, et al. Simulation training and its effect on long-term resident performance in central venous catheterization. Simul Healthc 2010;5:146-51. doi:10.1097/SIH.0b013e3181dd9672.

[7] Sturm LP, Windsor JA, Cosman PH, Cregan P, Hewett PJ, Maddern GJ. A systematic review of skills transfer after surgical simulation training. Ann Surg 2008;248:166-79. doi:10.1097/SLA.0b013e318176bf24.

[8] Scott DJ, Dunnington GL. The new ACS/APDS skills curriculum: Moving the learning curve out of the operating room. J Gastrointest Surg 2008;12:213-21.

doi:10.1007/s11605-007-0357-y.

[9] Bréaud J, Chevallier D, Benizri E, Fournier J-P, Carles M, Delotte J, et al. The place of simulation in the surgical resident curriculum. The pedagogic program of the Nice Medical School Simulation Center. J Visc Surg 2012;149:e52-60. doi:10.1016/j.jviscsurg.2011.12.007.

[10] ACS/APDS Surgery Resident Skills Curriculum n.d. https://www.facs.org/education/program/apds-resident (accessed January 4, 2016).

[11] Harris PA, Taylor R, Thielke R, Payne J, Gonzalez N, Conde JG. Research electronic data capture (REDCap)--a metadata-driven methodology and workflow process for providing translational research informatics support. J Biomed Inform 2009;42:377-81. doi:10.1016/j.jbi.2008.08.010.

[12] IBM Corp. Released. IBM SPSS Statistics for Windows, Version 20.0. 20112011.

[13] Male Urethral Catheterization — NEJM n.d. http://www.nejm.org/doi/full/10.1056/NEJMvcm054648 (accessed January 25, 2016).

[14] Kolozsvari NO, Feldman LS, Vassiliou MC, Demyttenaere S, Hoover ML. Sim one, do one, teach one: Considerations in designing training curricula for surgical simulation. $\mathrm{J}$ Surg Educ 2011;68:421-7. doi:10.1016/j.jsurg.2011.03.010. 


\begin{tabular}{|l|l|l|}
\hline Description & Clinical scenario & Unknown pathology \\
\hline Female trauma & $\begin{array}{l}\text { Motor vehicle accident, } \\
\text { pelvic fracture }\end{array}$ & Bladder injury \\
\hline Pre-op female & None & Labial constriction \\
\hline Pre-op male & $\begin{array}{l}\text { Rectal cancer, pre-operative } \\
\text { lower anterior resection }\end{array}$ & Complete obstruction \\
\hline Male, retention & $\begin{array}{l}\text { Benign Prostatic } \\
\text { Hypertrophy }\end{array}$ & None \\
\hline
\end{tabular}

Table 1 Description of the four simulated bladder catheterization procedures 


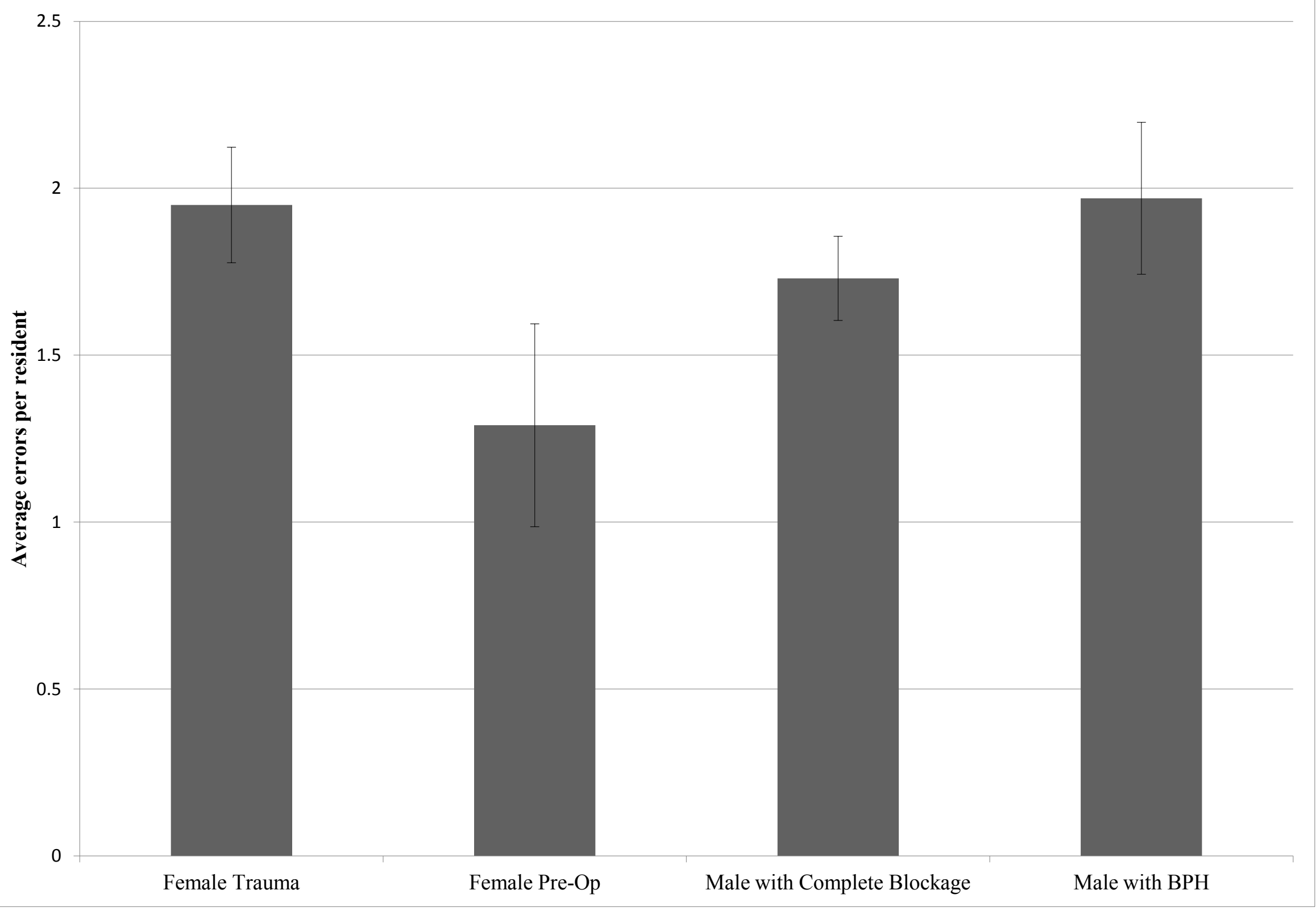




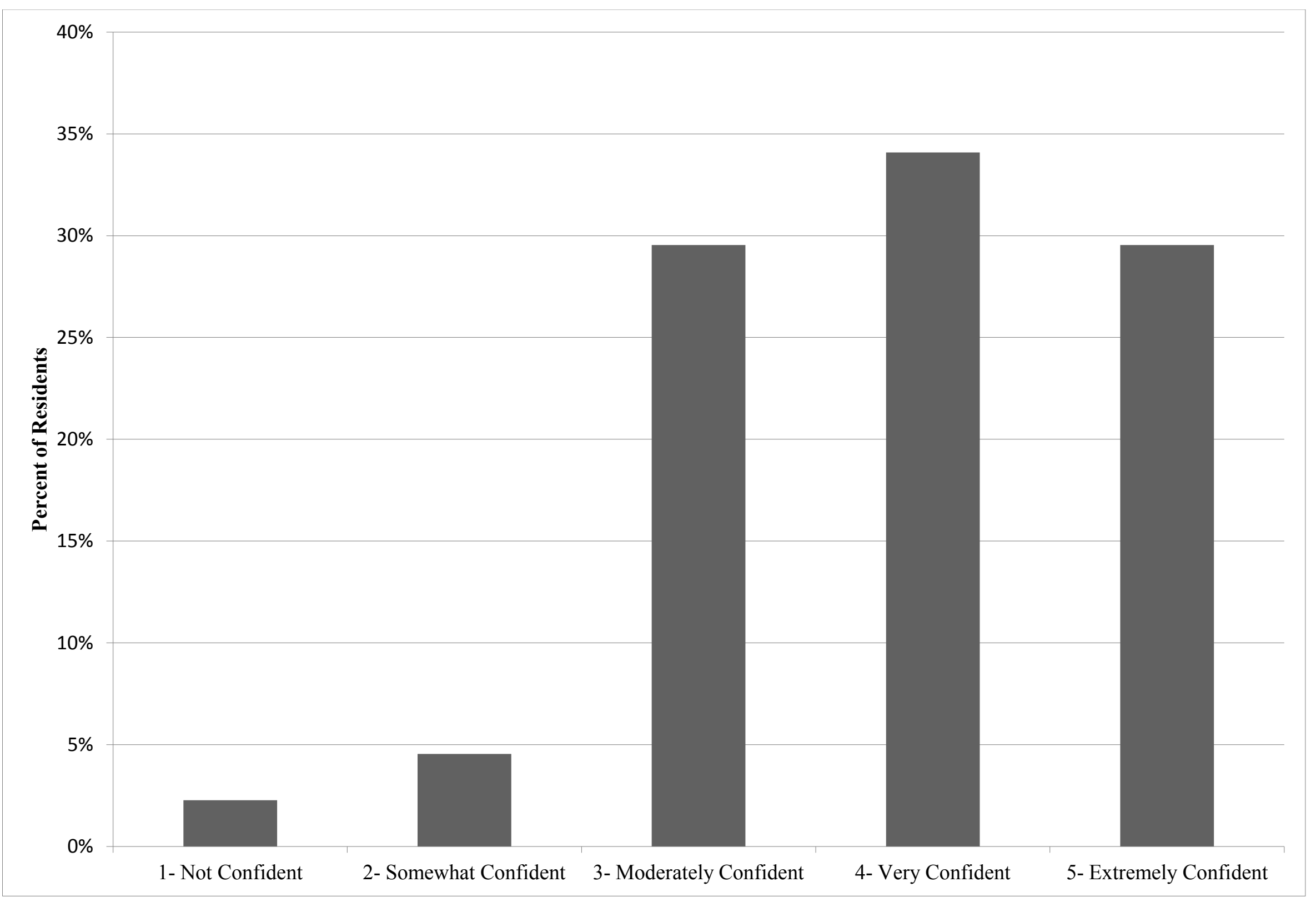




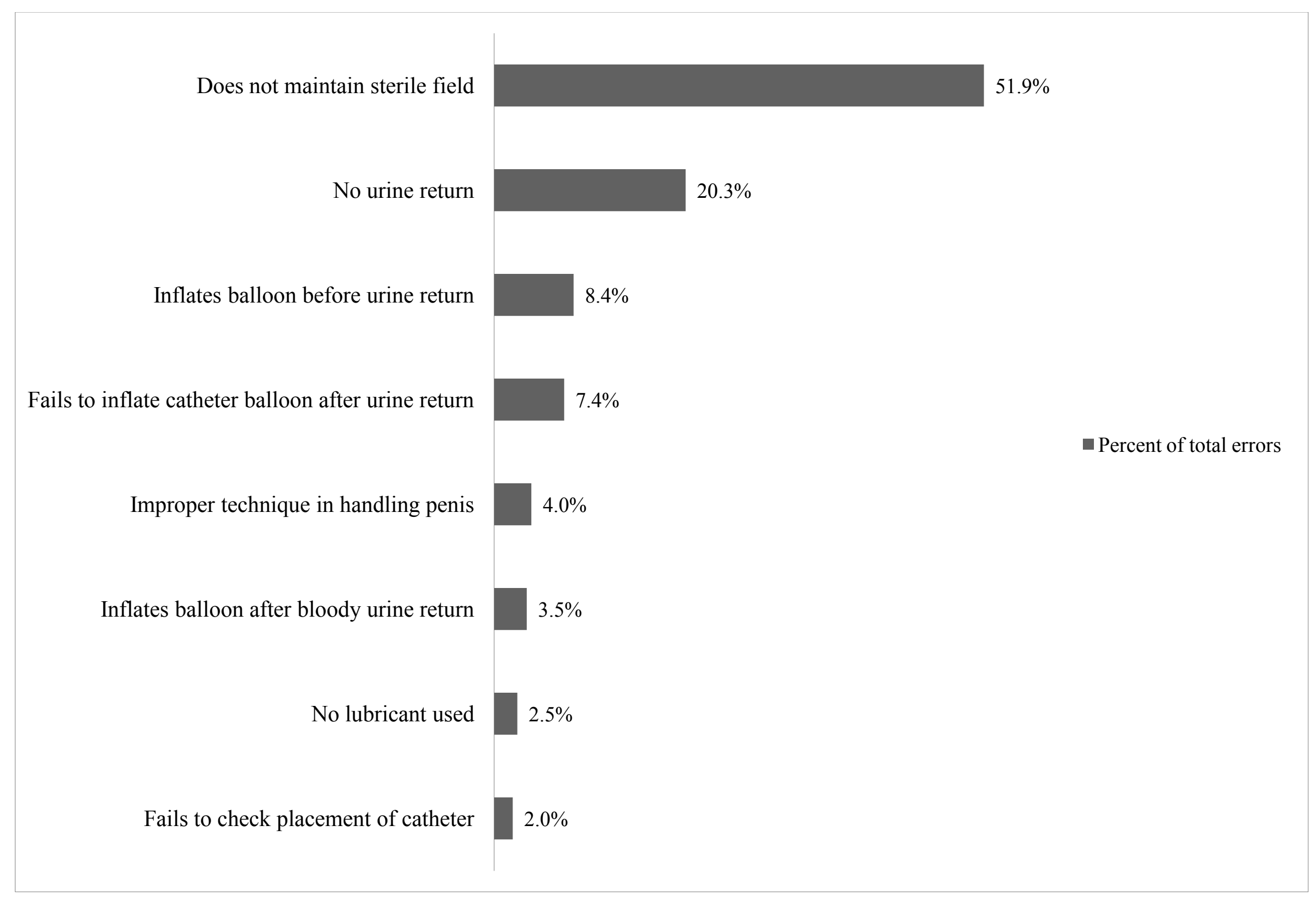


Does not maintain sterile field

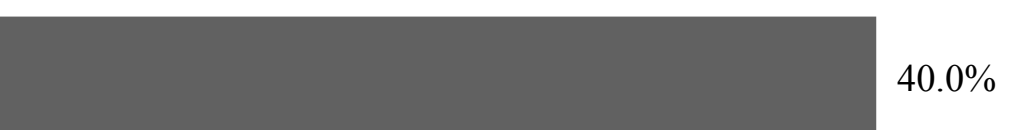

Inflates balloon after bloody urine return

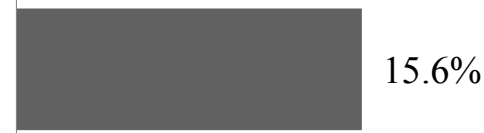

Inflates balloon before urine return

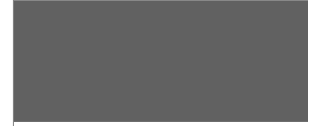

$13.3 \%$
Percent of residents

Fails to check placement of catheter after balloon inflation 
Figure 5

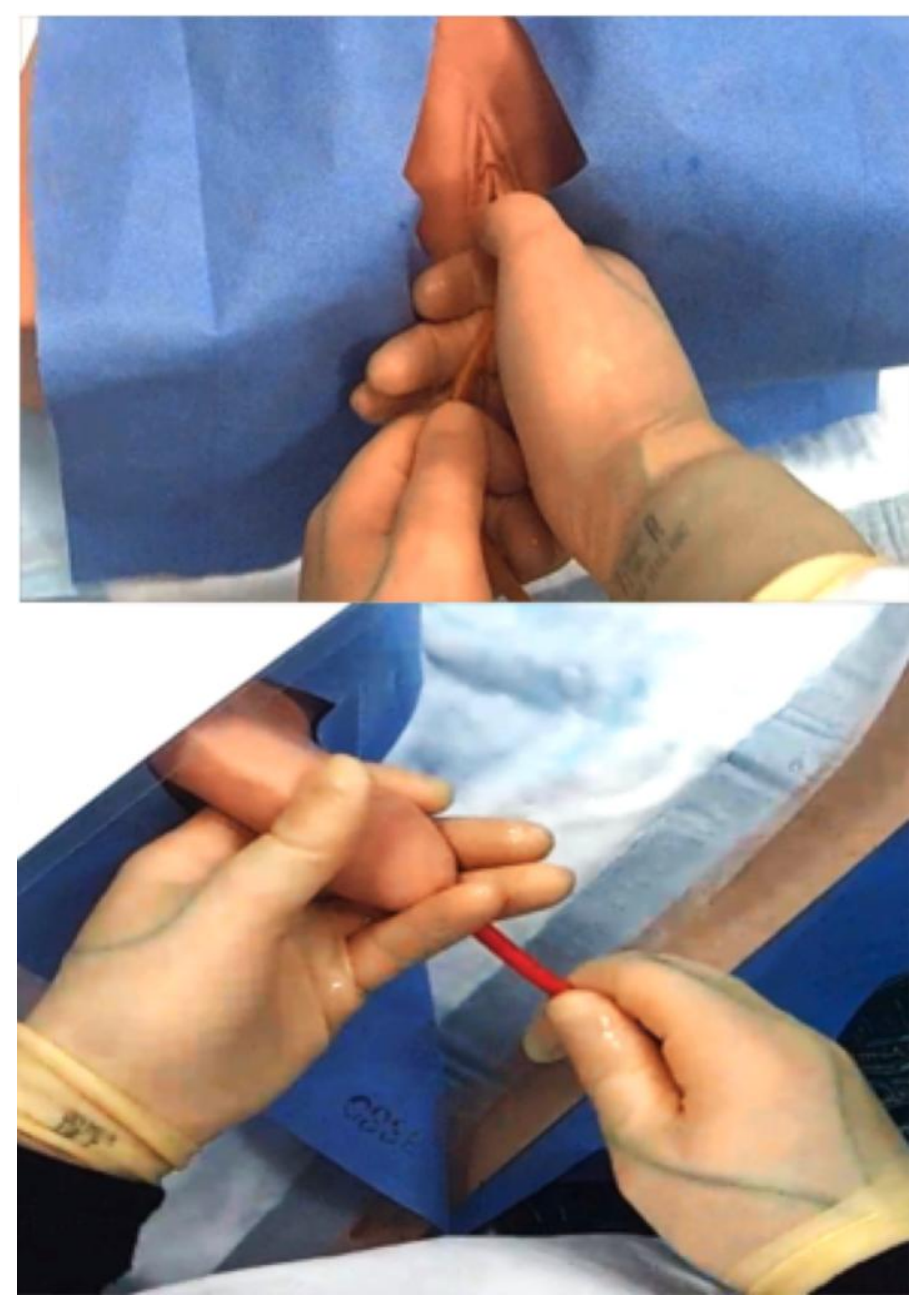

